\title{
Randomized Controlled Trial of a Parenting Program to Reduce the Risk of Child Maltreatment in South Africa
}

Lachman, Jaimie; Cluver, Lucy; Ward, Catherine L.; Hutchings, Judy; Mlotshwa, Sindisiwe; Wessels, Inge; Gardner, Frances

\section{Child Abuse and Neglect}

DOI:

10.1016/j.chiabu.2017.08.014

Published: 01/10/2017

Peer reviewed version

Cyswllt i'r cyhoeddiad / Link to publication

Dyfyniad o'r fersiwn a gyhoeddwyd / Citation for published version (APA):

Lachman, J., Cluver, L., Ward, C. L., Hutchings, J., Mlotshwa, S., Wessels, I., \& Gardner, F. (2017). Randomized Controlled Trial of a Parenting Program to Reduce the Risk of Child Maltreatment in South Africa. Child Abuse and Neglect, 72, 338-351.

https://doi.org/10.1016/j.chiabu.2017.08.014

\footnotetext{
Hawliau Cyffredinol / General rights

Copyright and moral rights for the publications made accessible in the public portal are retained by the authors and/or other copyright owners and it is a condition of accessing publications that users recognise and abide by the legal requirements associated with these rights.

- Users may download and print one copy of any publication from the public portal for the purpose of private study or research.

- You may not further distribute the material or use it for any profit-making activity or commercial gain

- You may freely distribute the URL identifying the publication in the public portal ?
}

Take down policy

If you believe that this document breaches copyright please contact us providing details, and we will remove access to the work immediately and investigate your claim. 


\section{Randomized Controlled Trial of a Parenting Program to Reduce the Risk of Child Mcontrolled trial of a parenting program to reduce the risk of child maltreatment in}

\section{South Africa}

Jamie M. Lachman ${ }^{\mathrm{a}, *}$

jamie.lachman@spi.ox.ac.uk, jamie.lachman@gmail.com

Lucie Cluver $^{\mathrm{a}, \mathrm{b}}$

Catherine L. Ward ${ }^{\mathrm{c}}$ d

Judy Hutchings ${ }^{\mathrm{e}}$

Sindisiwe Mlotshwa $^{\mathrm{f}}$

Inge M. Wessels $^{\mathrm{c}}$

Frances Gardner $^{\mathrm{a}}$

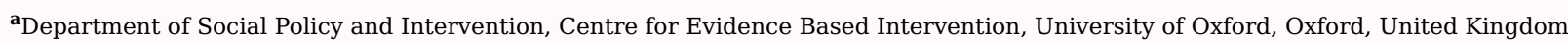

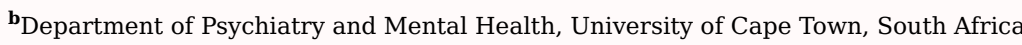

${ }^{\mathbf{c}}$ Department of Psychology, University of Cape Town, South Africa

dSafety and Violence Initiative, University of Cape Town, South Africa

eschool of Psychology, University of Wales Bangor, United KingdomCentre for Evidence Based Early Intervention, School of Psychology, Bangor University, Wales

f Department of Psychology, University of Witwatersrand, South Africa

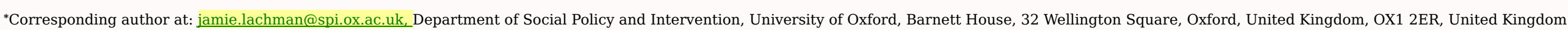

\section{Abstract}

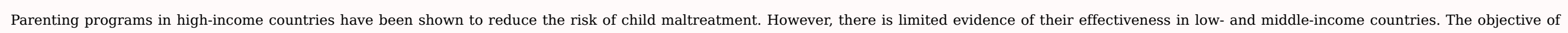

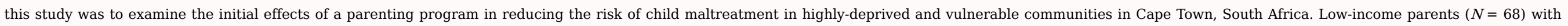

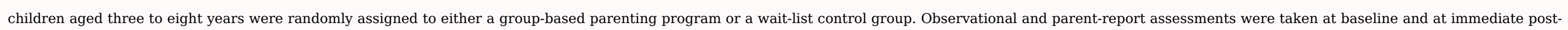

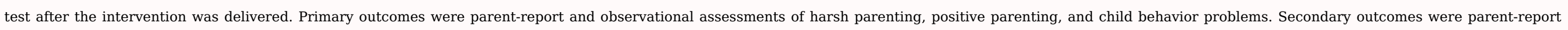

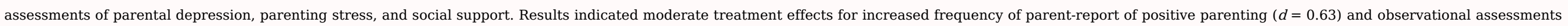

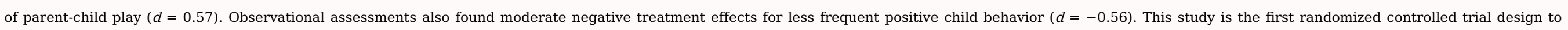

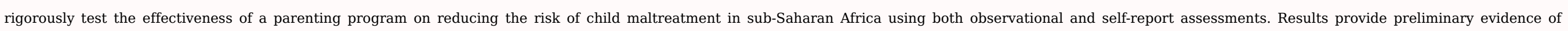

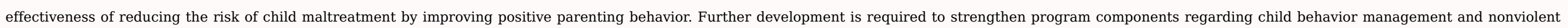
discipline strategies. Future research would benefit from a larger trial with sufficient power to determine program effectiveness. 


\section{Introduction}

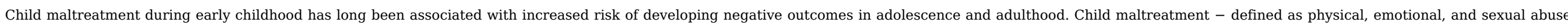

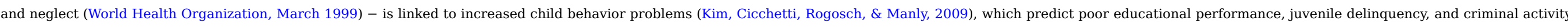

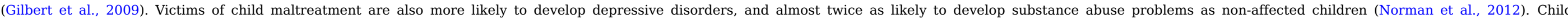

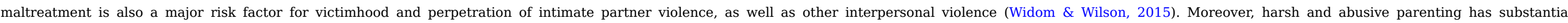

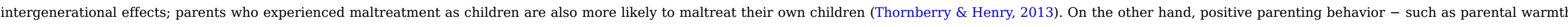
and consistent limit-setting - may reduce the likelihood of child maltreatment, especially in the context of cumulative risk (Trentacosta et al., 2008).

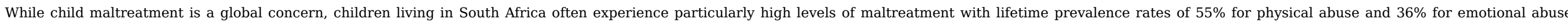

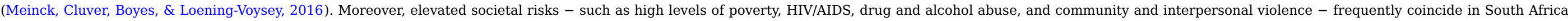

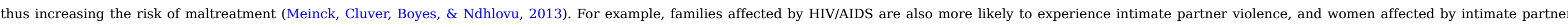

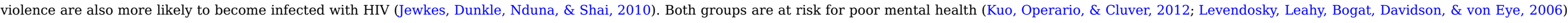

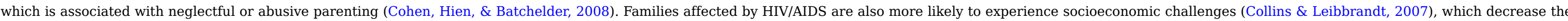

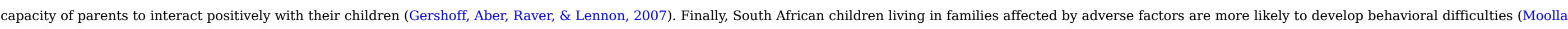
2012), which are reciprocally linked to increased risk of harsh parenting and potential maltreatment (Pardini, 2008).

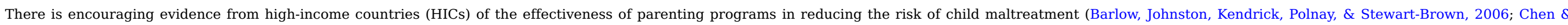

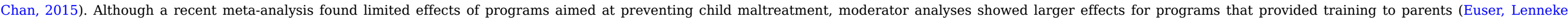

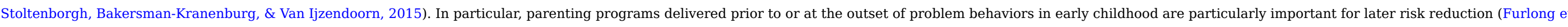

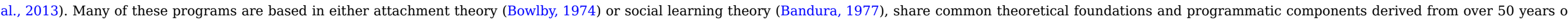

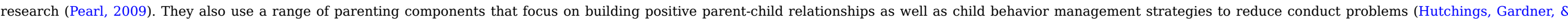

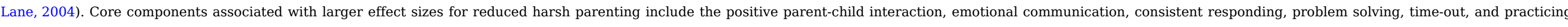

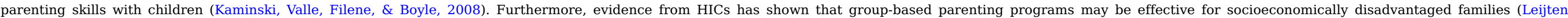
Raaijmakers, de Castro, \& Matthys, 2013; McGilloway et al., 2012).

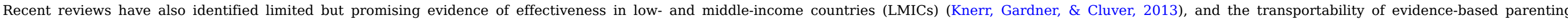

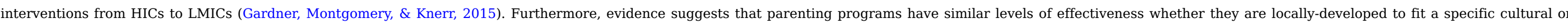

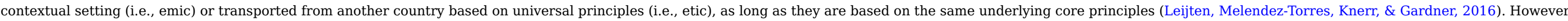

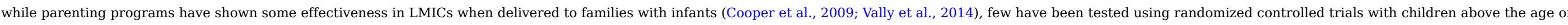
two years. In addition, a review of current group parenting programs implemented in South Africa found that few were based on evidence-based principles or components (Wessels \& Ward, 2015).

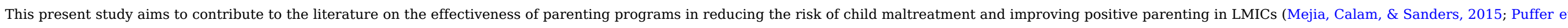

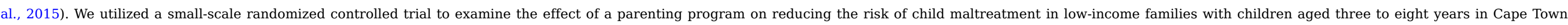

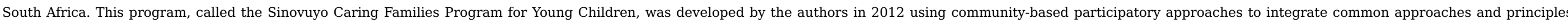

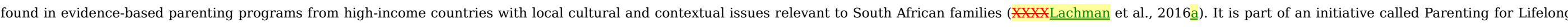

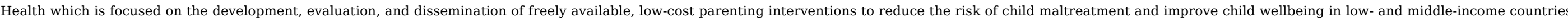

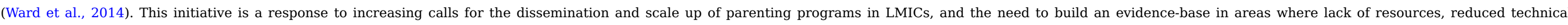
capacity, cultural differences, and increased adversity might compromise program effectiveness (Mejia, Leijten, Lachman, \& Parra-Cardona, 2016).

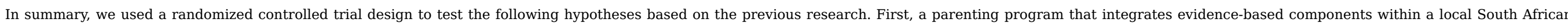

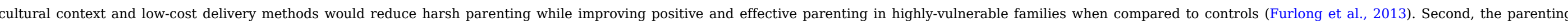




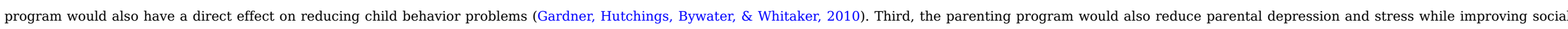

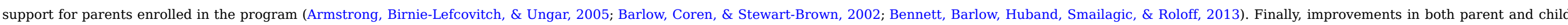
outcomes would contribute to the overall reduction in risk of child maltreatment (see Fig. 1).

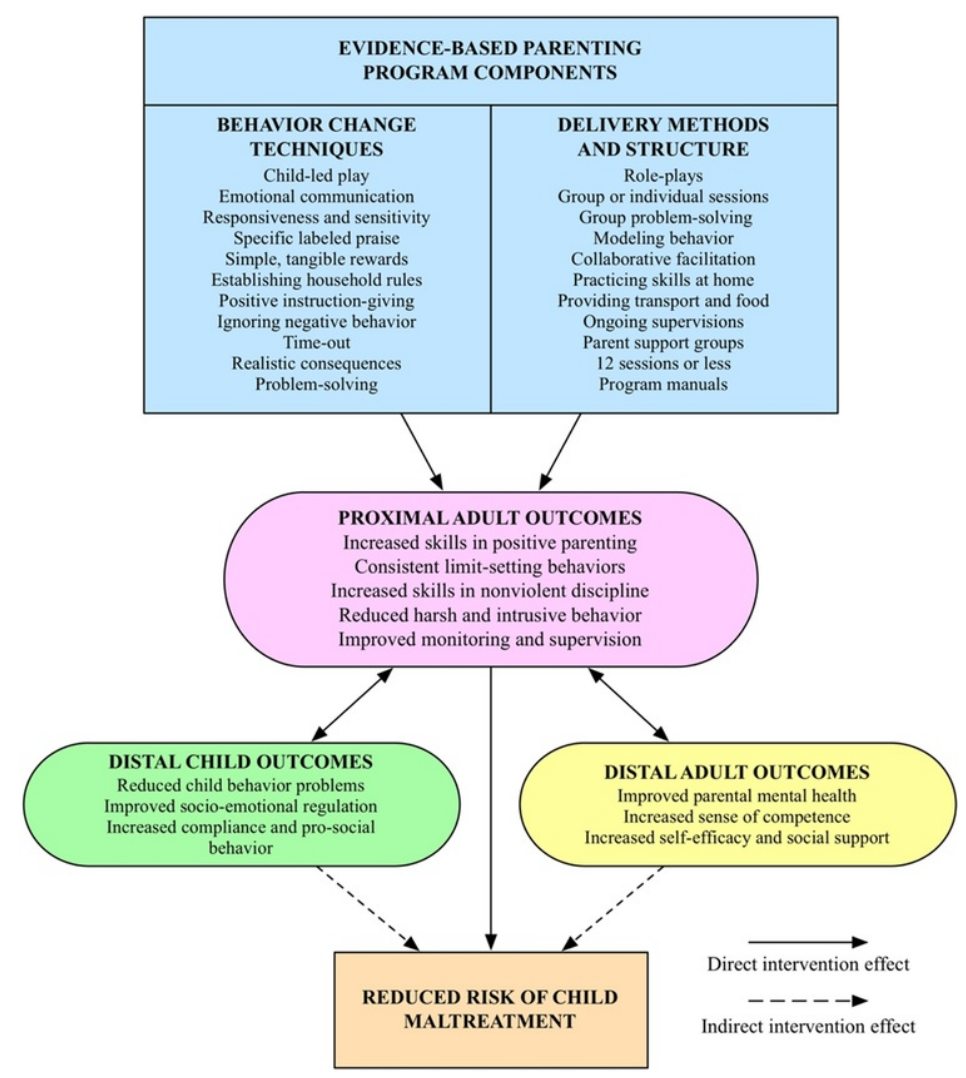

Fig. 1 XXXTheory of change model for parenting programs with reduced risk of child maltreatment as the ultimate outcome.

\section{alt-text. Fig. 1}

\section{Methods}

This trial is reported in line with the CONSORT (Consolidated Standards of Reporting Trials) guidelines on randomized controlled trials (Altman et al., 2001).

\subsection{Setting}

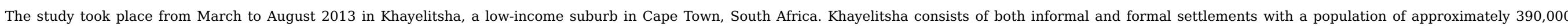

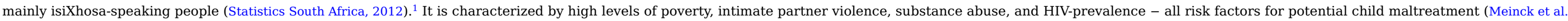
2013)

\subsection{Participants}

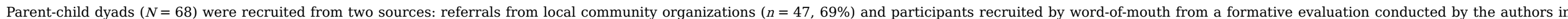




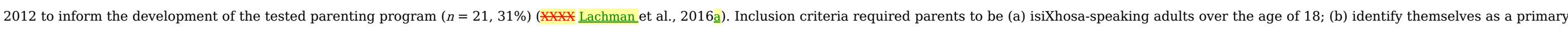

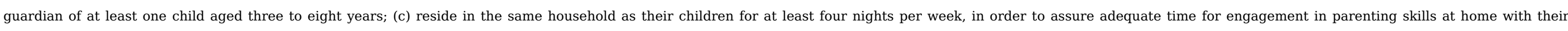

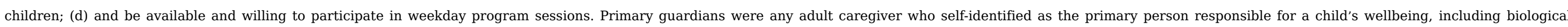

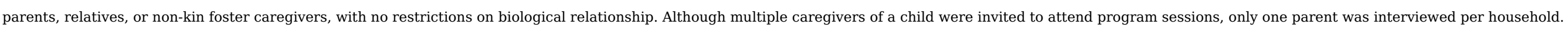

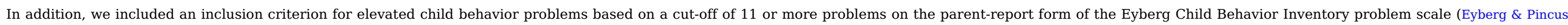

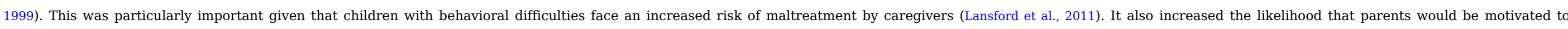

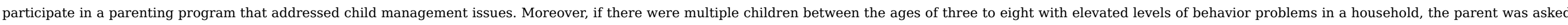
to choose the child whose behavior was the most difficult to manage. Finally, the exclusion criteria included participants or children who exhibited acute mental health problems or severe disabilities.

\subsection{Power calculations}

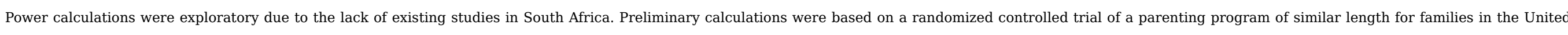

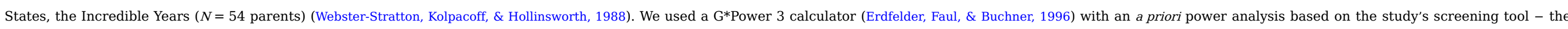

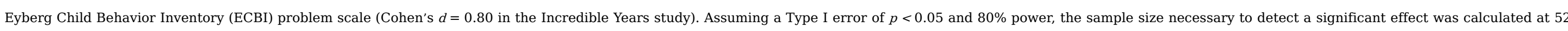

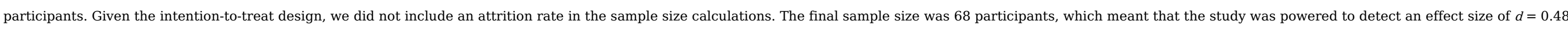
or greater.

\subsection{Randomization}

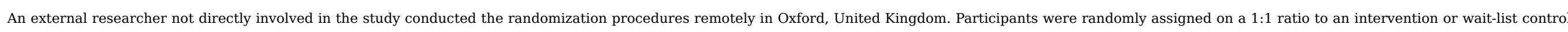

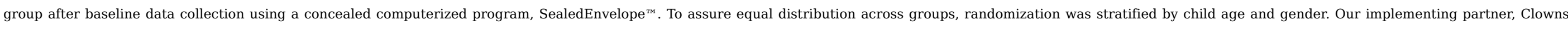

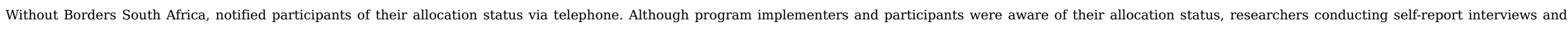
observational assessments were blind to allocation. After post-test data collection, the control group received the intervention from September to November 2013.

\subsection{Ethical procedures}

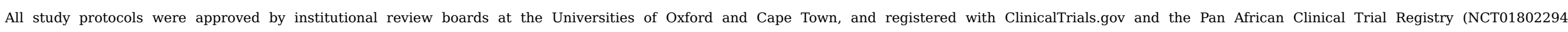

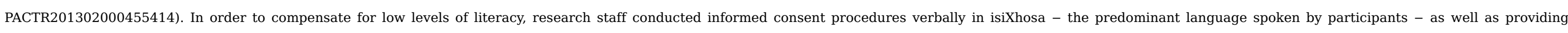

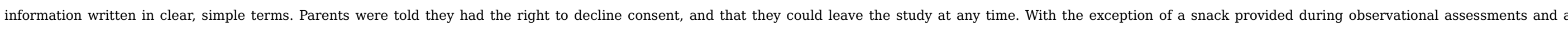

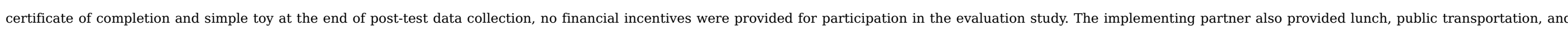
certificates to parents during parenting sessions as part of program delivery.

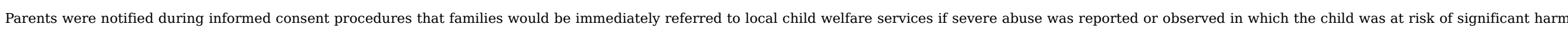

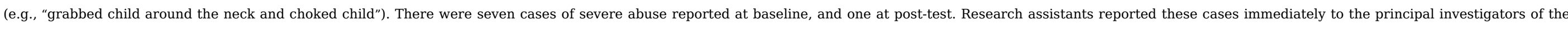
study who then made referrals.

\subsection{Intervention}

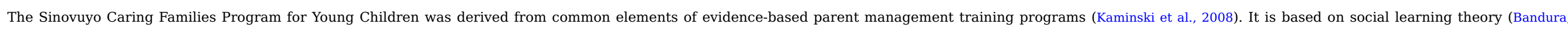

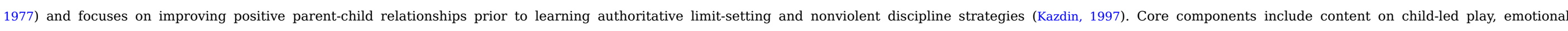

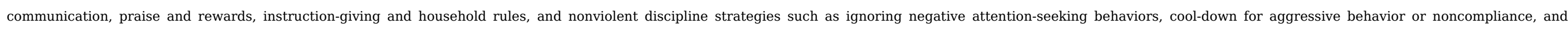
consequences (Hutchings et al., 2004).

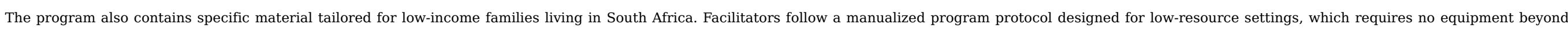




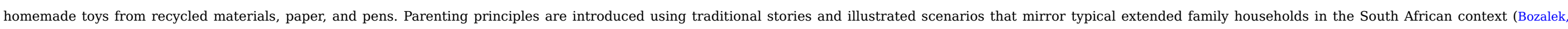

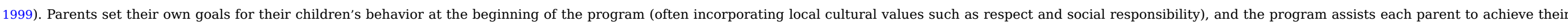

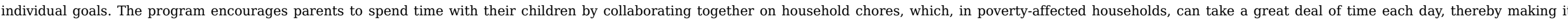

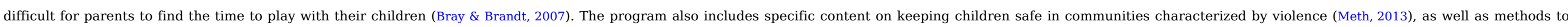

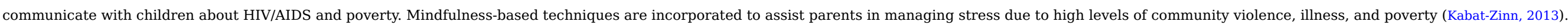

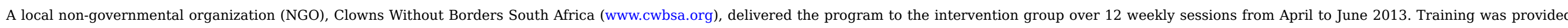

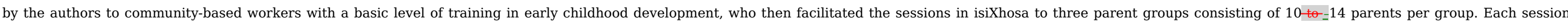

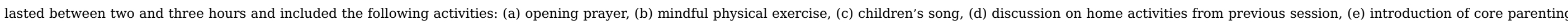

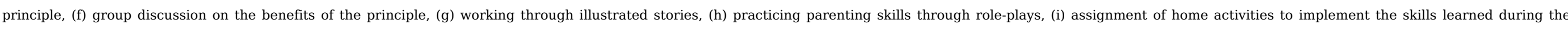
session, and (j) closing prayer. Whenever possible, one-on-one home consultations were provided to parents who missed a session (less than one home consultation per session)(Fable 1).

Table 1 Demographic characteristics of the sample at baseline. ${ }^{1}$

alt-text: Table 1

Control $(n=34)$

Intervention $(n=34)$

$p$ value

Family characteristics

Informal housing, $n$ (\%)

$25,73.5 \%$

$26,76.5 \%$

$\underline{0.784}$

Household size, M (SD)

5.56 (2.19)

5.58 (3.43)

$\underline{0} .441$

Parent characteristics

Parent age, M (SD)

Parent gender, $n$ female, \%

Marital status, $n$ single, $\%$

Not completed high school, $n$ (\%)

Unemployed, $n$ (\%)

41.09 (13.32)

42.06 (13.16)

…606

$34,100.0 \%$

$33,97.1 \%$

1.000

$26,76.5 \%$

$24,70.6 \%$

0.784

$25,73.5 \%$

$31,90.9 \%$

$\underline{0} .109$

Child characteristics

Child age, M (SD)

$32,94.1 \%$

$34,100.0 \%$

$\underline{0} .493$

Child gender, $n$ female (\%)

$5.18(1.73)$

$5.62(1.65)$

$\underline{0} .798$

$17,50.0 \%$

$16,47.1 \%$

1.000

Relationship to parent, $n$ biological (\%)

$20,58.8 \%$

$21,61.8 \%$

1.000

Social risk factors for child abuse

Experienced hunger $\geq 5$ times in previous 30 days, ${ }^{\mathrm{a}} n$ (\%)

$25,73.5 \%$

$29,85.3 \%$

$\underline{0} .369$

Family affected by HIV/AIDS, ${ }^{\mathrm{b}} n$ (\%)

$11,32.4 \%$

$10,29.4 \%$

1.000

Parent experienced intimate partner violence in previous month, ${ }^{\mathrm{c}} n(\%)$

$12,35.3 \%$

$11,32.4 \%$

1.000

Parent experienced physical abuse as a child, ${ }^{\mathrm{d}} n(\%)$

$24,70.6 \%$

25, $73.5 \%$

…624 
$\underline{1} \underline{\text { Independent } t} t$-tests and Chi-squared tests found no significant differences between groups at baseline.

a Hunger Scale Questionnaire.

b Three or more symptoms on the Verbal Autopsy.

c Conflict Tactics Scale.

d ISPCAN Child Abuse Screening Tool-Retrospective.

\subsection{Measures}

\subsubsection{Demographic information}

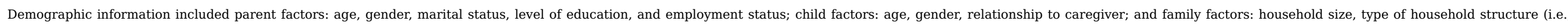

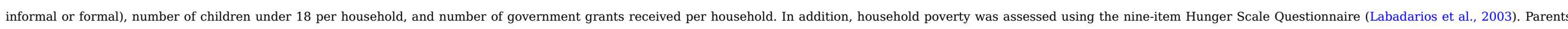

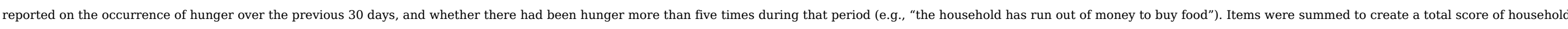
hunger intensity.

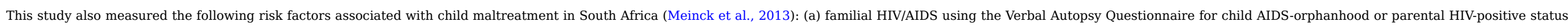

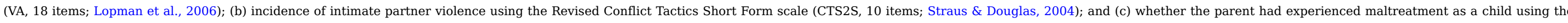

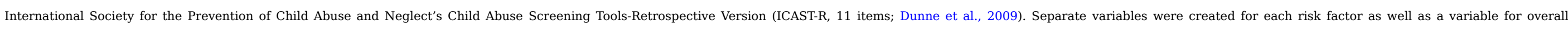
cumulative risk for child maltreatment that included household poverty.

\subsubsection{Primary intervention outcomes}

Primary outcomes associated with increased risk of child maltreatment were self-report and observational assessments of harsh parenting, positive parenting, and child behavior problems.

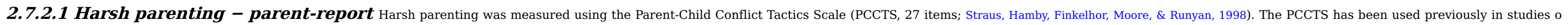

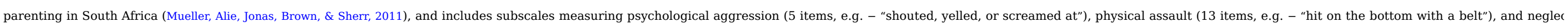

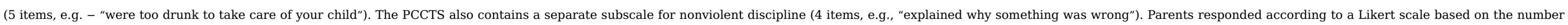

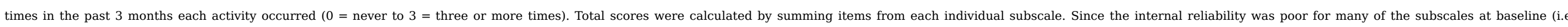

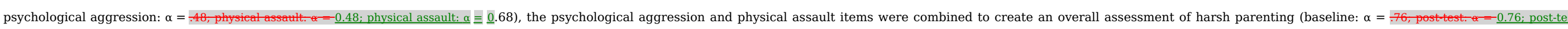

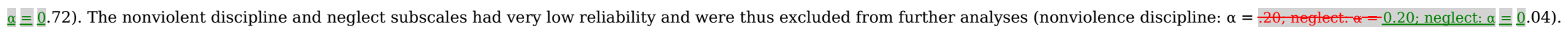

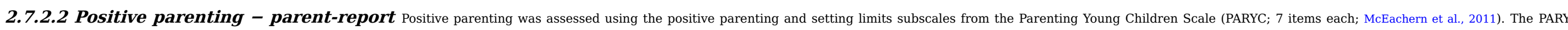

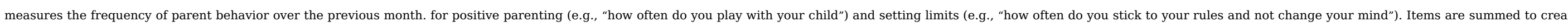

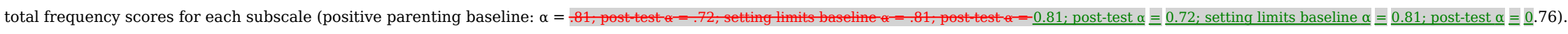

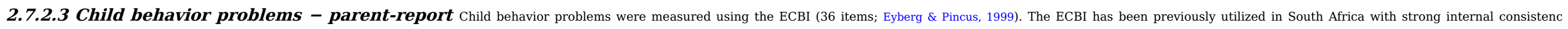

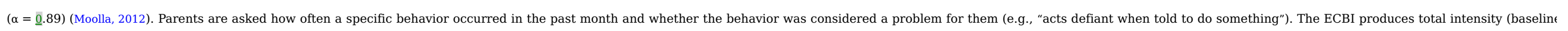

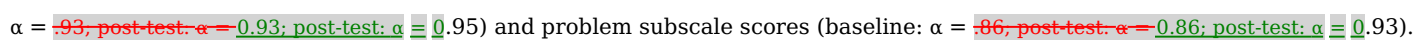

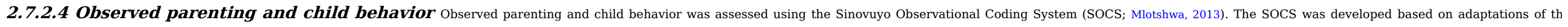

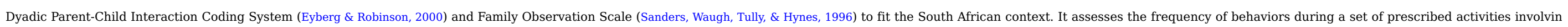

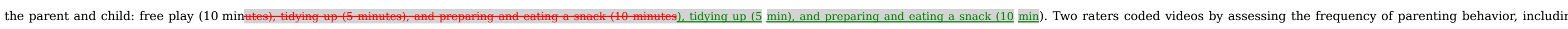

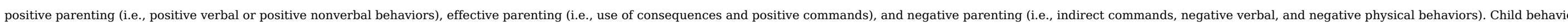




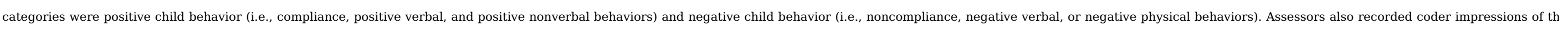

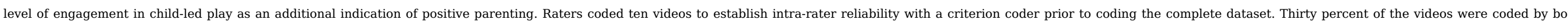

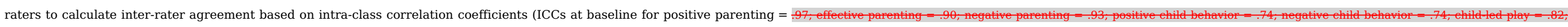

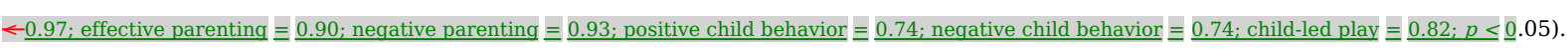

\subsubsection{Secondary outcomes}

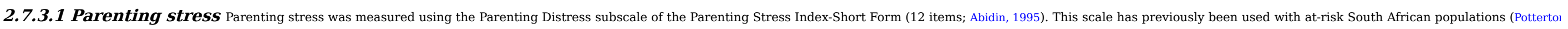

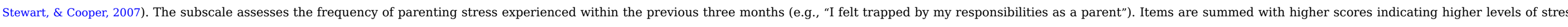
(baseline: $\alpha=.85$, post-test- $\alpha=.73) .2 .7 .3 .2$ Parental depression. 0.85 ; post-test: $\alpha \equiv \underline{0.73 \text { ). }}$

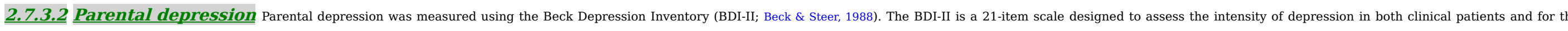

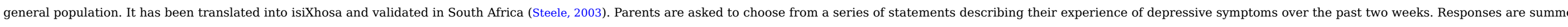
with higher scores indicating higher levels of depressive symptoms (baseline: $\alpha=0$;

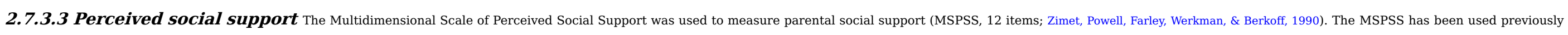

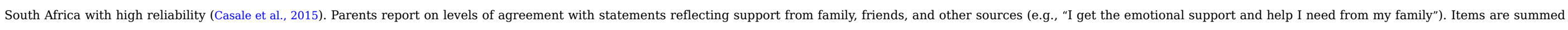
create a total score with higher scores indicating higher levels of perceived social support (baseline: $\alpha=70$,

\subsection{Data collection and management}

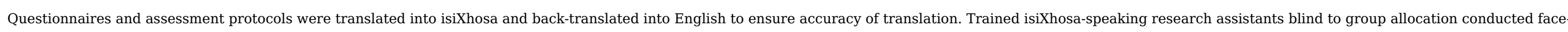

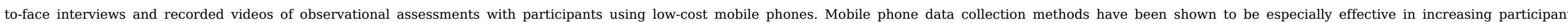

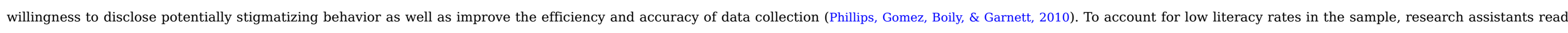
out loud the informed consent information as well as each item and associated choice option on the questionnaire.

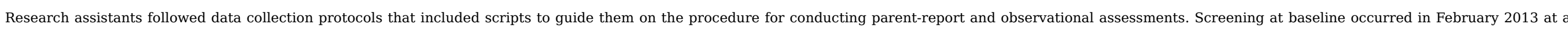

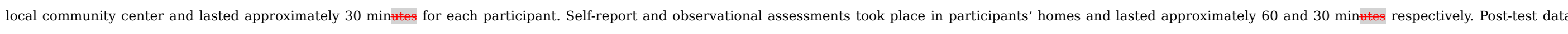
collection took place in July 2013, immediately after the intervention group received the program, and about three and a half months after baseline.

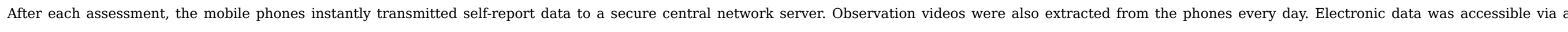

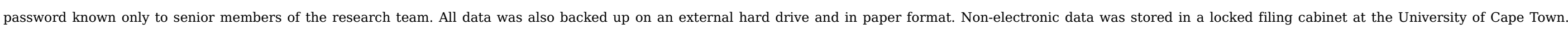
Outcome data were cleaned and entered into SPSS 21.0 for analysis.

\subsection{Data analysis}

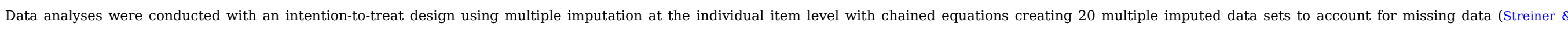

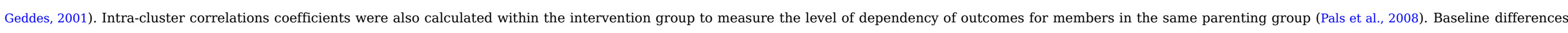

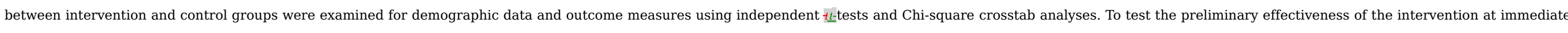

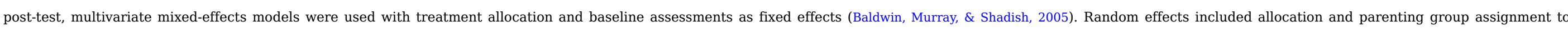

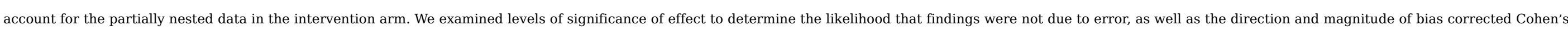

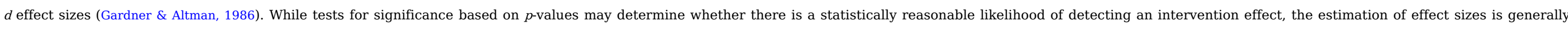

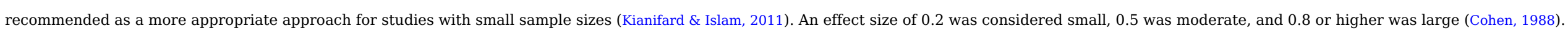

\section{Results}




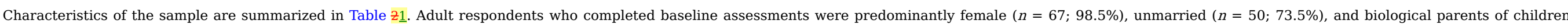

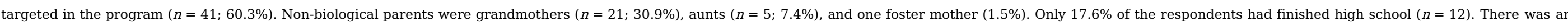

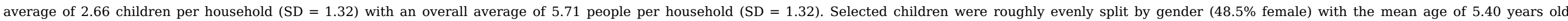
$(\mathrm{SD}=1.69)$.

Table 2 Results summarizing primary outcomes using multivariate mixed modeling and an intention-to-treat analysis.

\section{alt-text: Table 2}

\begin{tabular}{|c|c|c|c|c|c|c|c|}
\hline & \multicolumn{2}{|c|}{ Control $(n=34)$} & \multicolumn{2}{|c|}{ Intervention $(n=34)$} & \multirow[t]{2}{*}{ F Statistic } & \multirow[t]{2}{*}{ Estimated mean difference ${ }^{2}[95 \% \mathrm{CI}]$} & \multirow[t]{2}{*}{ Effect size $^{3}[95 \% \mathrm{CI}]$} \\
\hline & Baseline & Post-test ${ }^{1}$ & Baseline & Post-test ${ }^{1}$ & & & \\
\hline Measure & $\mathrm{M}(\mathrm{SD})$ & $\mathrm{M}(\mathrm{SD})$ & $\mathrm{M}(\mathrm{SD})$ & $\mathrm{M}(\mathrm{SD})$ & & & \\
\hline \multicolumn{8}{|l|}{ Harsh parenting } \\
\hline Harsh parenting, parent-reporta & $9.95(8.06)$ & $3.47(3.01)$ & $8.24(5.75)$ & $3.55(5.18)$ & 0.01 & $0.08[-1.98,2.14] 0.02[-1.98,2.14]$ & $\underline{0.02[-0.46,0.49]}$ \\
\hline Negative parenting, observed ${ }^{b}$ & $5.59(5.11)$ & $4.53(3.34)$ & $7.48(9.70)$ & $3.32(3.78)$ & 2.03 & $1.21+[2.94,0.52]-1.21 \pm[-2.94,0.52]$ & $=0.34+[-0.81,0.14]$ \\
\hline \multicolumn{8}{|l|}{ Positive parenting } \\
\hline Positive parenting, parent-reportc & $20.12(9.30)$ & $20.21(7.28)$ & $21.50(9.09)$ & $25.29(8.56)$ & $6.97^{*}$ & $5.08^{* *}[1.23,8.92]$ & $0.63^{* *}[0.14,1.12]$ \\
\hline Positive parenting, observed ${ }^{b}$ & $7.71(8.93)$ & $6.63(5.56)$ & $7.78(6.33)$ & $7.40(5.56)$ & 0.34 & $0.77[-2.09,3.63] 0.13[-2.09,3.63]$ & $\underline{0.13[-0.35,0.61]}$ \\
\hline Child-led play, observed ${ }^{b}$ & $0.54(0.72)$ & $0.45(0.73)$ & $0.64(0.73)$ & $0.87(0.70)$ & $5.70^{*}$ & $0.42^{*}[0.07,0.76]$ & $0.57^{*}[0.09,1.06]$ \\
\hline Setting limits, parent-reportc & 23.79 (8.77) & $21.12(7.06)$ & $24.79(9.30)$ & $20.71(8.71)$ & 0.06 & $0.41[4.25,3.43]=0.41[-4.25,3.43]$ & $=0.05[-0.53,0.42]$ \\
\hline Effective parenting, observed ${ }^{b}$ & $13.06(9.39)$ & $8.66(4.55)$ & $10.62(5.27)$ & $8.21(3.95)$ & 0.20 & $-0.45[2.51,1.62]=0.45[-2.51,1.62]$ & $=0.10[-0.58,0.37]$ \\
\hline \multicolumn{8}{|l|}{ Child behavior } \\
\hline Intensity, parent-report ${ }^{\mathrm{d}}$ & $122.56(42.50)$ & $111.39(37.72)$ & $120.59(41.66)$ & $99.78(41.85)$ & 1.44 & $11.59[30.88,7.70]=11.59[-30.88,7.70]$ & $=0.29[-0.77,0.19]$ \\
\hline Problem, parent report ${ }^{\mathrm{d}}$ & $21.09(7.06)$ & $16.45(7.98)$ & $20.74(6.87)$ & $15.49(9.52)$ & 0.21 & $-0.97[5.22,3.29]-0.97[-5.22,3.29]$ & $=0.11[-0.58,0.37]$ \\
\hline Positive behavior, observed ${ }^{b}$ & $17.95(11.72)$ & $13.52(7.21)$ & $15.48(9.82)$ & $9.99(5.05)$ & $5.60^{*}$ & $-3.53 *[6.54,0.52]-3.53 *-[-6.54,-0.52]$ & $=0.56^{*}[-1.05,-0.08]$ \\
\hline Negative behavior, observed ${ }^{b}$ & $2.24(7.00)$ & $0.52(1.66)$ & $1.85(1.96)$ & $1.08(2.36)$ & $2.46^{+}$ & $0.56[-0.34,1.45] 0.30[-0.34,1.45]$ & $\underline{0.30[-0.18,0.78]}$ \\
\hline
\end{tabular}

${ }^{1}$ Post-test means adjusted for baseline scores.

${ }^{2}$ Difference in mean follow-up scores between intervention and waiting list control conditions adjusted for baseline scores.

${ }^{3}$ Bias-corrected Cohen's $d$.

${ }^{*} \mathrm{p}<.05 . *{ }^{*} \mathrm{p}<.01 .1 \mathrm{p}<\underline{0.05}$.

$\stackrel{* *}{\underline{p}} \leq \underline{0.01}$.

$\pm \underline{p} \leq \underline{0} .15$.

a Parent-Child Conflict Tactics Scale. 
b Sinovuyo Observational Coding System.

c Parenting Young Children Scale.

d Eyberg Child Behavior Inventory.

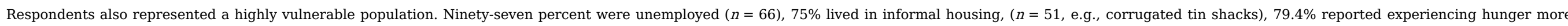

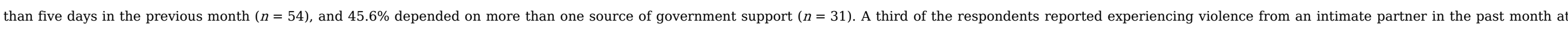

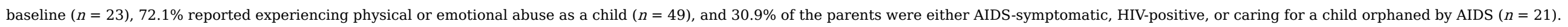

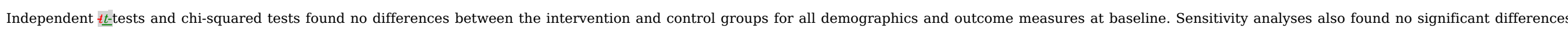

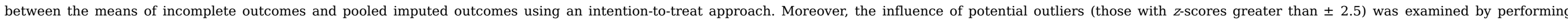

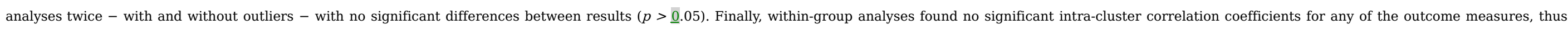
indicating that there was minimal effect of parent group assignment within the intervention allocation.

\subsection{Study flow}

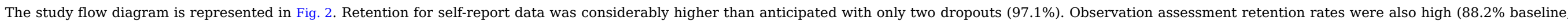

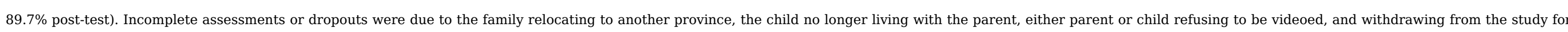

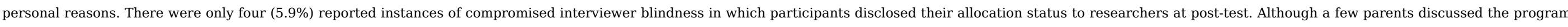
with control group participants who lived nearby, significant contamination was considered unlikely based on interviews with the control group.

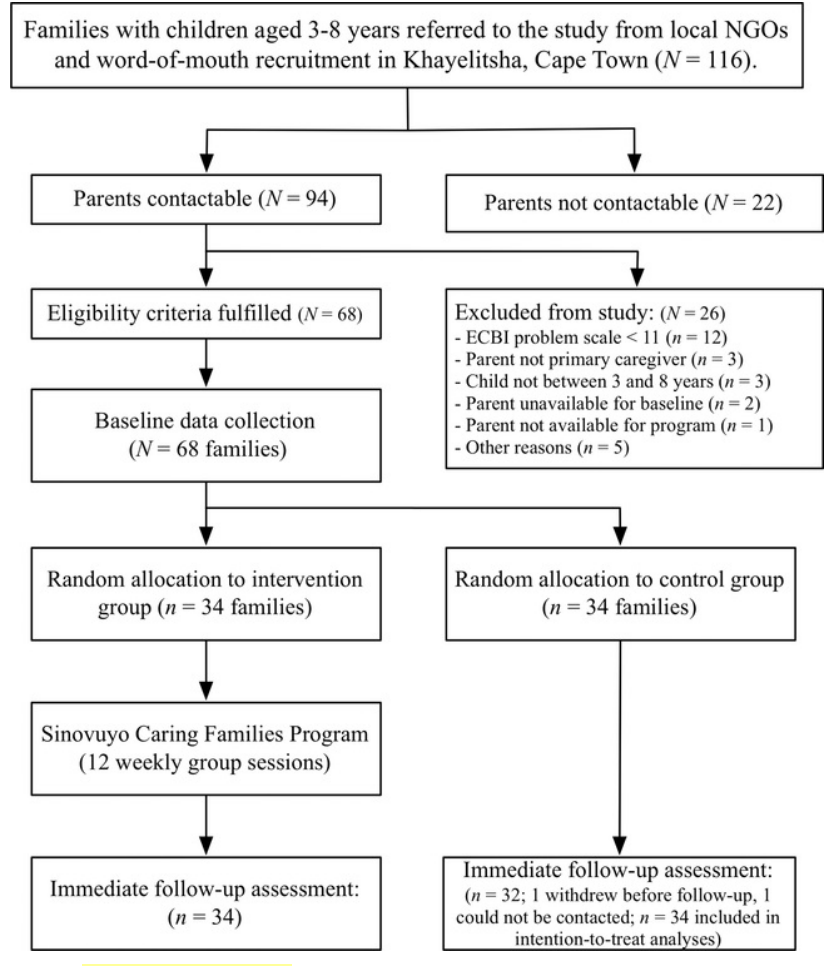

Fig. 2 Consort diagram. 


\subsection{Treatment of missing data}

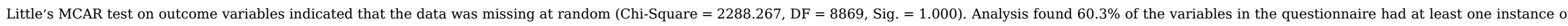

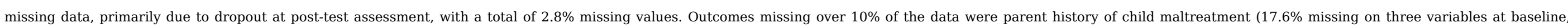
$16.2 \%$ missing on eight variables at post-test) and observation assessments of parenting and child behavior (11.8\% and $10.3 \%$ missing variables at baseline and post-test, respectively).

\subsection{Program participation and fidelity}

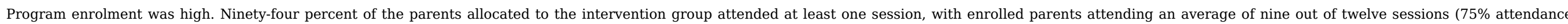

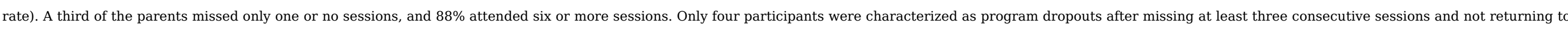
the program. Facilitators also reported implementing $91 \%$ of the manualized activities based on fidelity checklists.

\subsection{Outcomes}

Multivariate mixed model analyses for parenting and child behavior outcomes are summarized in Table 3.

Table 3 Results summarizing secondary outcomes using multivariate mixed modeling and an intention-to-treat analysis.

\begin{tabular}{|c|c|c|c|c|c|c|c|}
\hline \multirow[b]{2}{*}{ Measure } & \multicolumn{2}{|c|}{ Control $(n=34)$} & \multicolumn{2}{|c|}{$\begin{array}{l}\text { Intervention } \\
\quad(\mathrm{n}=34)\end{array}$} & \multirow[t]{2}{*}{$\begin{array}{c}F \\
\text { Statistic }\end{array}$} & \multirow[t]{2}{*}{ Estimated mean difference 2 [95\% CI] } & \multirow[t]{2}{*}{$\begin{array}{c}\text { Effect size }^{3} \\
{[95 \% \mathrm{CI}]}\end{array}$} \\
\hline & Baseline & Post-test $^{1}$ & Baseline & $\begin{array}{l}\text { Post- } \\
\text { test }^{1}\end{array}$ & & & \\
\hline & M (SD) & $\mathrm{M}(\mathrm{SD})$ & $\mathrm{M}(\mathrm{SD})$ & M (SD) & & & \\
\hline \multicolumn{8}{|l|}{ Parent Behavior } \\
\hline $\begin{array}{l}\text { Parenting } \\
\text { stress }^{a}\end{array}$ & $\begin{array}{l}24.20 \\
(9.79)\end{array}$ & $\begin{array}{l}22.40 \\
(7.63)\end{array}$ & $\begin{array}{l}21.90 \\
(9.17)\end{array}$ & $\begin{array}{l}20.53 \\
(8.20)\end{array}$ & 0.96 & $1.88[5.72,1.96]-1.88[-5.72,1.96]$ & $\begin{array}{l}=0.23[-0.71 \\
0.24]\end{array}$ \\
\hline $\begin{array}{l}\text { Parental } \\
\text { depression }\end{array}$ & $\begin{array}{l}14.60 \\
(10.41)\end{array}$ & $\begin{array}{l}8.77 \\
(8.59)\end{array}$ & $\begin{array}{l}12.36 \\
(10.78)\end{array}$ & $\begin{array}{l}9.93 \\
(10.02)\end{array}$ & 0.26 & $\begin{array}{l}1.16[3.36,5.68] 0.12[0.35,0.60] \text { Social supporte } 58.60(9.70) 57.41(10.04) 55.65(9.55) 60.07(9.38) 1.252 .63[2.07,7.34] 0.27[0.21, \\
\theta .75] * p<.05 ; * k p<.01 ; 1 p<-3.36,5.68]\end{array}$ & $\underline{0.12[-0.35}$ \\
\hline$\underline{\text { Social support }}^{\complement}$ & $\frac{58.60}{(9.70)}$ & $\underline{57.44}$ & $\frac{55.65}{(9.55)}$ & $\frac{60.07}{(9.38)}$ & $\underline{1.25}$ & $\underline{2.63[-2.07,7.34]}$ & $\underline{0.27[-0.21,}$ \\
\hline
\end{tabular}

\section{${ }_{-\underline{p}}^{*} \leq \underline{0.05 ;}{ }^{* *} \underline{\underline{p}} \leq \underline{0.01 ;}{ }^{ \pm} \underline{\underline{p}} \leq \underline{0} .10$.}

${ }^{1}$ Post-test means adjusted for baseline scores.

${ }^{2}$ Difference in mean follow-up scores between intervention and waiting list control conditions adjusted for baseline scores.

${ }^{3}$ Bias corrected Cohen's $d$

a Parenting Stress Index-Short Form.

b Beck Depression Inventory.

c Multidimensional Scale of Perceived Social Support.

\subsubsection{Parenting}




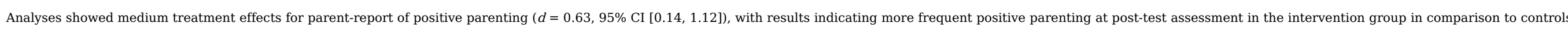

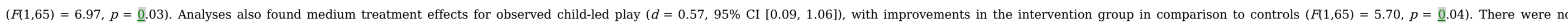

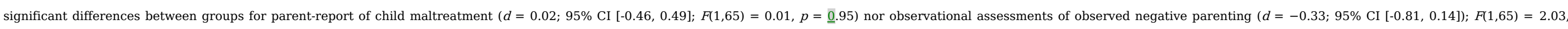
$p=\underline{0} .17)$.

\subsubsection{Child behavior}

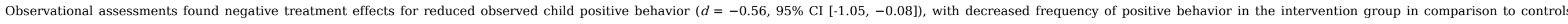

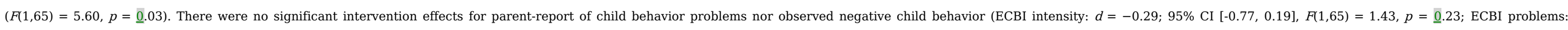
$d=-0.11 ; 95 \%$ CI [-0.58, 0.37], F(1,65) $=0.20, p=\underline{0} .68$; observed negative behavior: $d=0.30 ; 95 \%$ CI $[-0.18,0.78], F(1,65)=3.47, p=\underline{0} .07)$.

\subsubsection{Secondary outcomes}

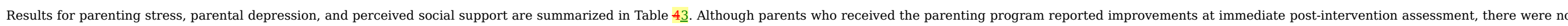

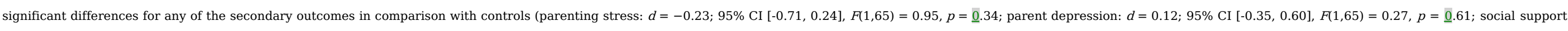
$d=0.27 ; 95 \%$ CI [-0.21, 0.75], $F(1,65)=1.23, p=\underline{0} .28)$.

\section{Discussion}

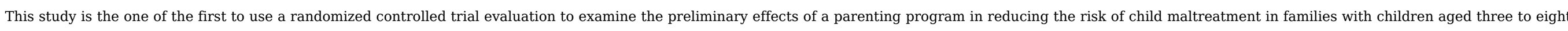

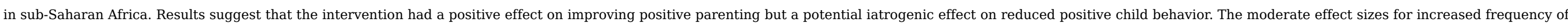

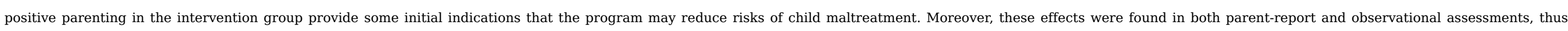

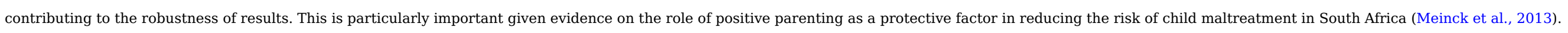

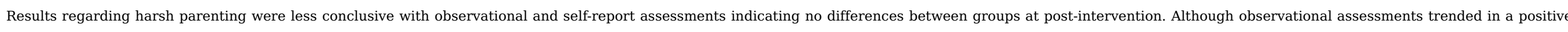

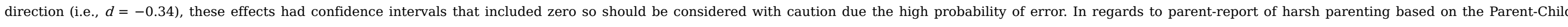

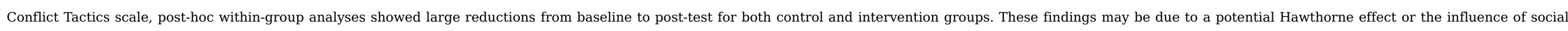
desirability as a result of participating in a study focused on reducing child maltreatment (Flay, 1986).

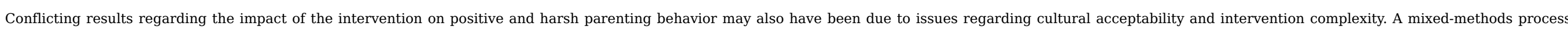

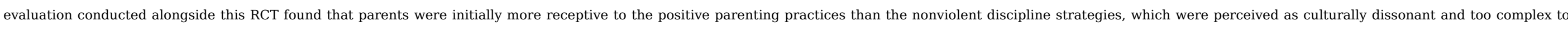

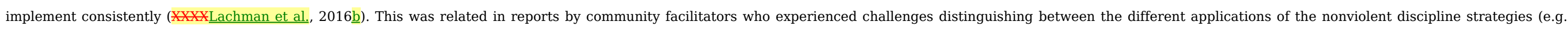

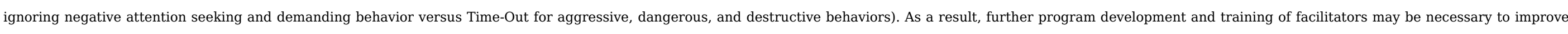
competency of delivery while strengthening the cultural acceptability of nonviolent discipline components as a replacement behavior to harsh parenting.

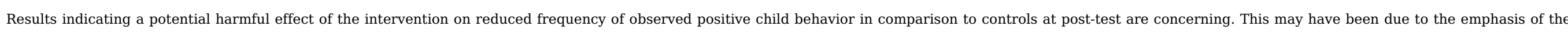

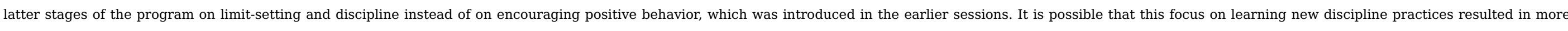

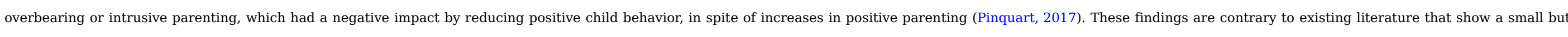

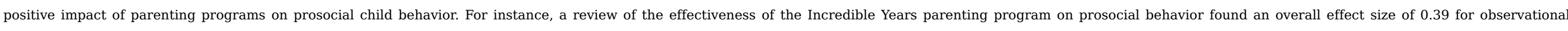

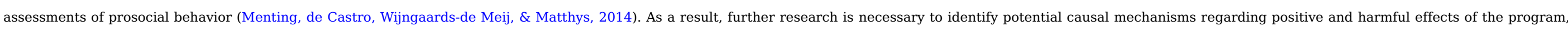

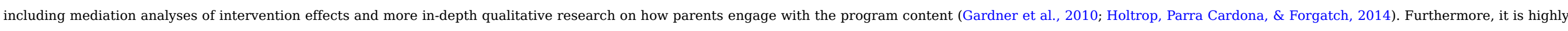

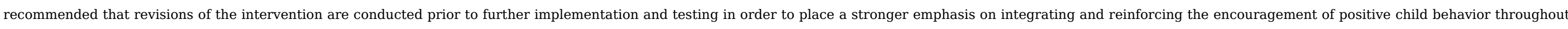
the program. 


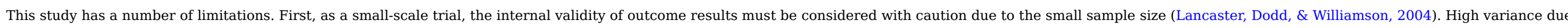

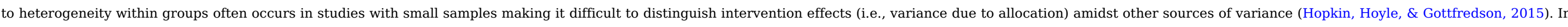

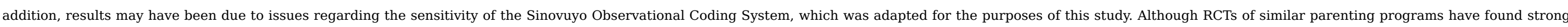

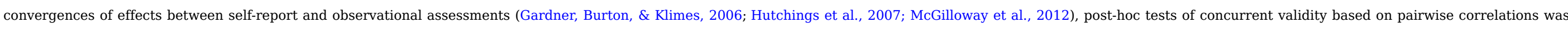
not established between observational and self-report measures of child behavior.

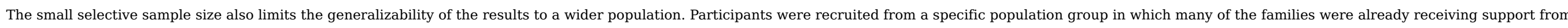

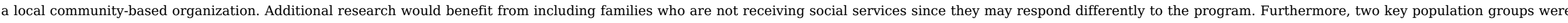

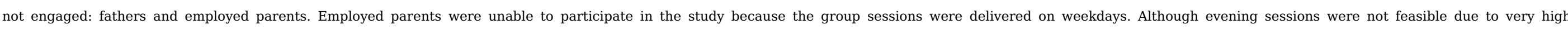

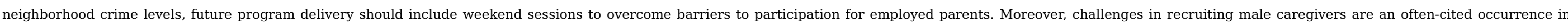

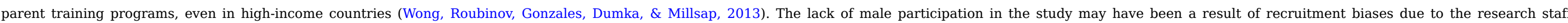

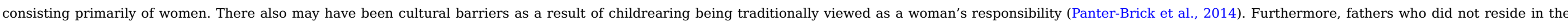

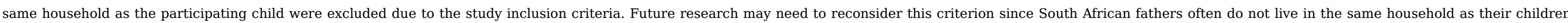
and have limited contact with them over the course of their lifetimes (Bray, Gooskens, Kahn, Moses, \& Seekings, 2010).

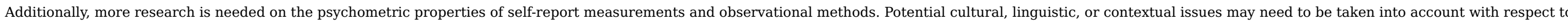

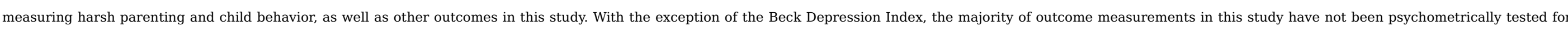

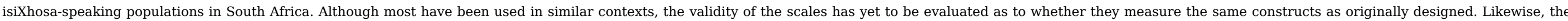
Sinovuyo Observational Coding System may require additional piloting and testing to determine its reliability in assessing parent and child behaviors.

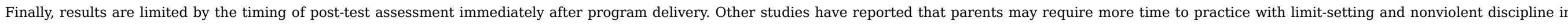

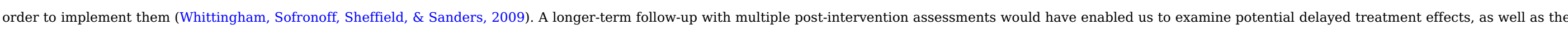
possible mediation between positive parenting, child behavior, and child maltreatment (Kumkale \& Albarracin, 2004).

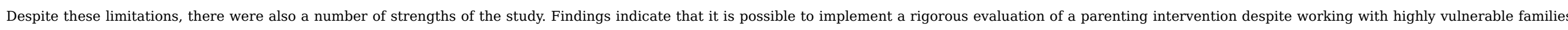

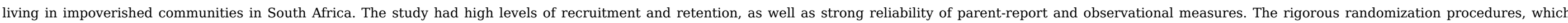

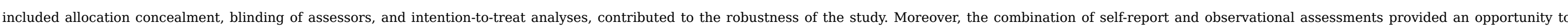
analyze intervention effects from multiple perspectives, thus diminishing potential reporting biases due to social desirability.

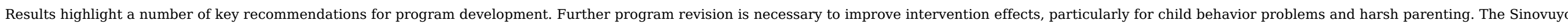

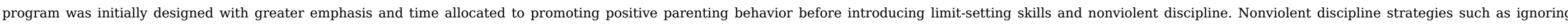

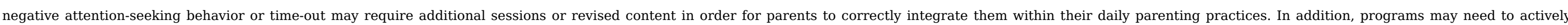
involve parents and children in joint sessions or via intensive coaching at home in order to improve child behavior.

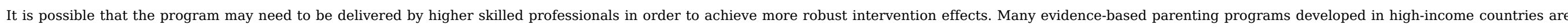

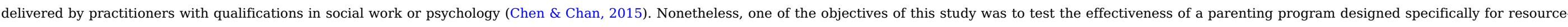

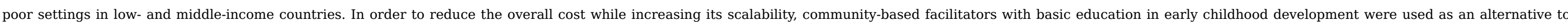

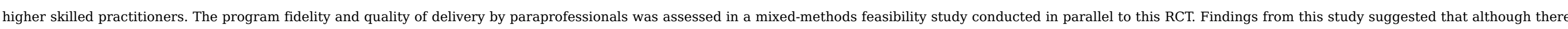

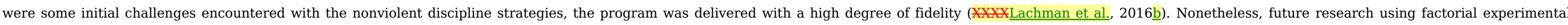
designs is recommended in order to explore the differential effects of delivering parenting programs in LMICs by higher- or lower-skilled practitioners.

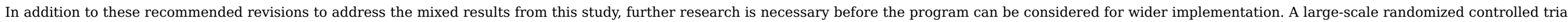

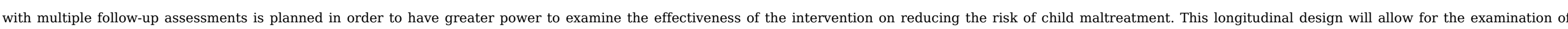




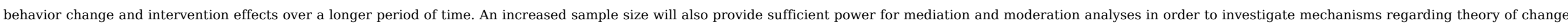
models as well as potential differential effects for specific characteristics of the population (Kraemer, Wilson, Fairburn, \& Agras, 2002; Wang \& Ware, 2011).

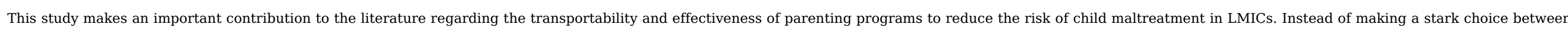

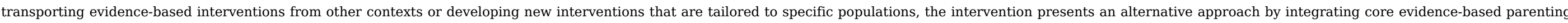

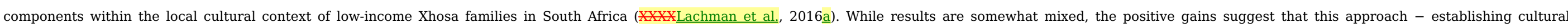

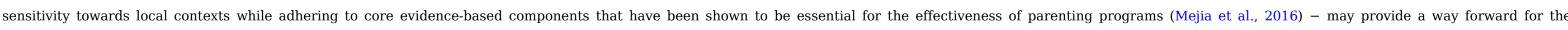
implementation of parenting programs in LMICs.

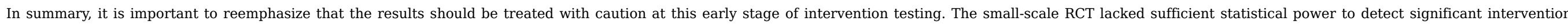

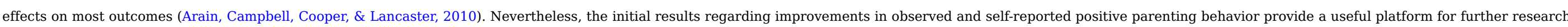

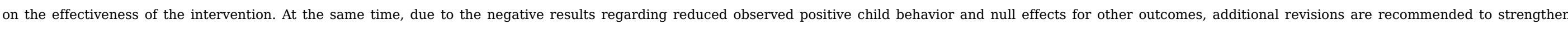
program content and delivery prior to subsequent testing and implementation.

\section{Author Nnote}

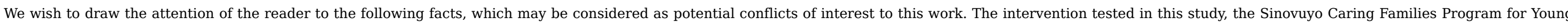

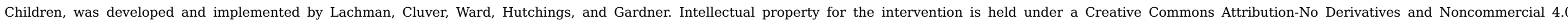

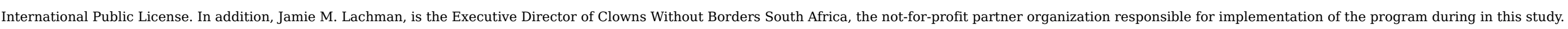

\section{Funding}

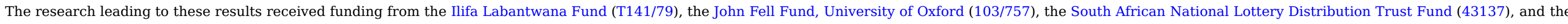
European Research Council under the European Union's Seventh Framework Programme (FP7/2007-2013; ERC grant agreement 313421).

\section{References}

Abidin R.R., Parenting Stress Istress index (PSI) manual, 3rd ed., 1995, Pediatric Psychology Press; Charlottesville, VA.

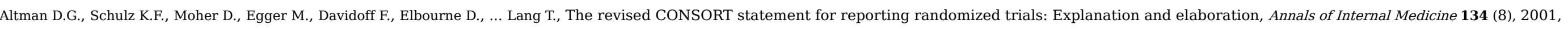
663-694.

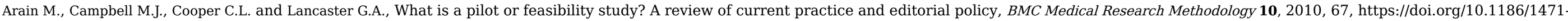
2288-10-67.

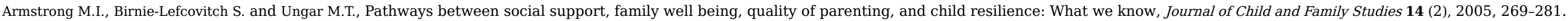

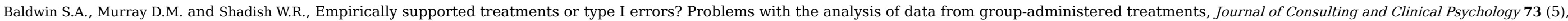

2005, 924-935, https://doi.org/10.1037/0022-006x.73.5.924.

Bandura A., Social learning theory, 1977, General Learning Press; New York.

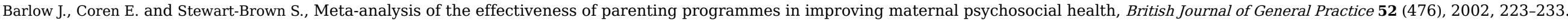

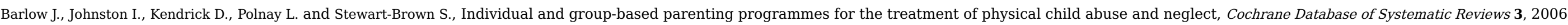
$1-20$.

Beck A.T. and Steer R.A., Psychometric properties of the Beck Depression Inventory: Twenty-five years of evaluations, Clinical Psychology Review 8, 1988, 77-100. 


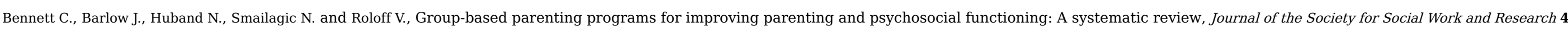

(4), 2013, 300-332.

Bowlby J., Attachment and loss Vol. 1, 1974, Hogarth Press and Institute of Psycho-Analysis; London.

Bozalek V., Contextualizing caring in black South African families, Social Politics 6 (1), 1999, 85-99.

Bray R. and Brandt R., Child care and poverty in South Africa, Journal of Children and Poverty 13 (1), 2007, 1-19, https://doi.org/10.1080/10796120601171187.

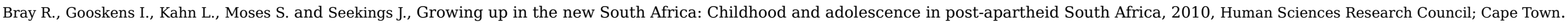

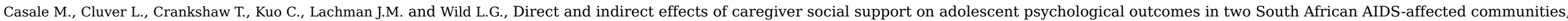

American Journal of Community Psychology 2015, 1-11.

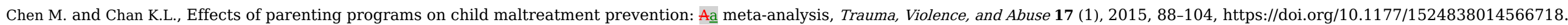

Cohen L.R., Hien D.A. and Batchelder S., The impact of cumulative maternal trauma and diagnosis on parenting behavior, Child Maltreatment 13 (1), $2008,27-38$.

Cohen J., Statistical power analysis for the behavioral sciences, 1988, Erlbaum; Hillsdale, NJ.

Collins D. and Leibbrandt M., The financial impact of HIV/AIDS on poor households in South Africa, AIDS 21 (Suppl. 7), 2007, S75-S81.

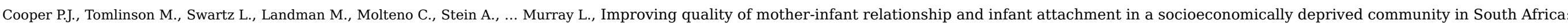

Randomised controlled trial, British Medical Journal 338, 2009, b974.

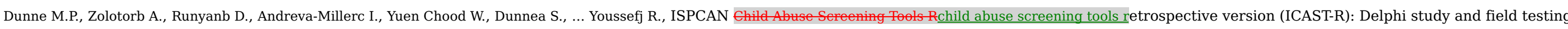
in seven countries, -Child Abuse \&Child Abuse \& Neglect 33 (11), 2009, 815-825.

Erdfelder E., Faul F. and Buchner A., GPOWER: A general power analysis program, Behavior Research Methods, Instruments, and Computers: A Journal of the Psychonomic Society, Inc 28, $1996,1-11$.

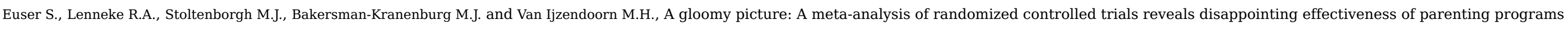
aiming at preventing child maltreatment, BMC Public Health 15 (1), 2015, 1068-1082, https://doi.org/10.1186/s12889-015-2387-9.

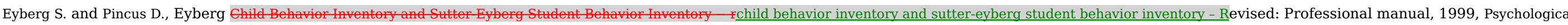
Assessment Resources; Odessa, FL.

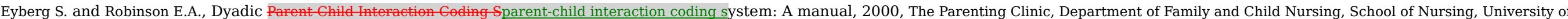
Washington; Seattle, WA.

Flay B.R., Efficacy and effectiveness trials (and other phases of research) in the development of health promotion programs, Preventive Medicine 15 (5), $1986,451-474$.

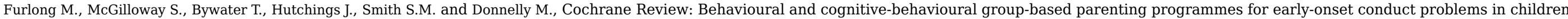
aged 3to=12 years (Review), Evidence-Based Child Health: A Cochrane Review Journal 8 (2), 2013, 318-692, https://doi.org/10.1002/ebch.1905.

Gardner M.J. and Altman D.G., Confidence-intervals rather than P-values: Estimation rather than hypothesis-testing, British Medical Journal 292 (6522), 1986, 746-750.

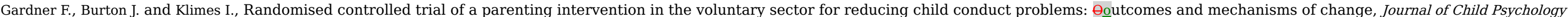
and Psychiatry 47 (11), 2006, 1123-1132, https://doi.org/10.1111/J.1469-7610.2006.01668.X.

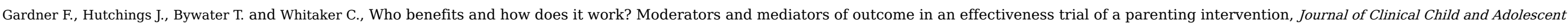
Psychology 39 (4), 2010, 568-580, https://doi.org/10.1080/15374416.2010.486315. 


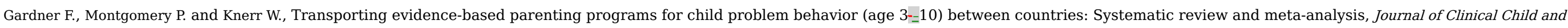
Adolescent Psychology 2015, 1-14, https://doi.org/10.1080/15374416.2015.1015134.

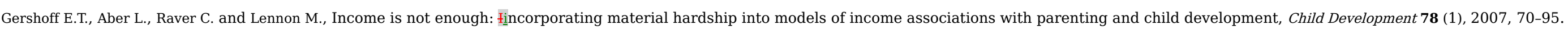

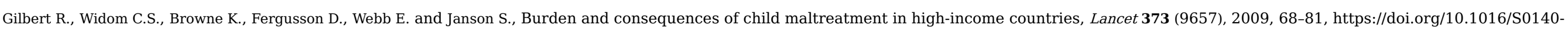
6736(08)61706-7.

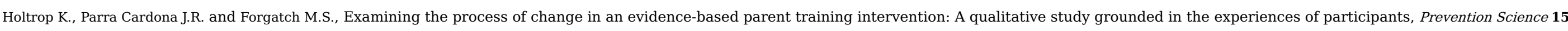
(5), 2014, 745-756, https://doi.org/10.1007/S11121-013-0401-Y.

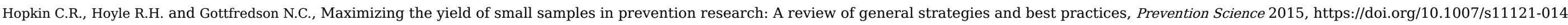
0542-7.

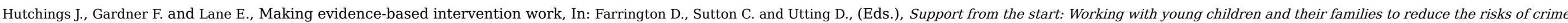
and antisocial behaviour, 2004, Department for Education and Skills; London, I(pp. 69-79).

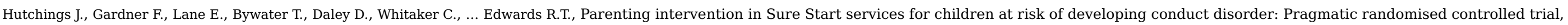
British Medical Journal 334 (7595), 2007, 678-682, https://doi.org/10.1136/Bmj.39126.620799.55.

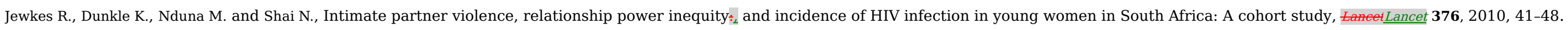

Kabat-Zinn J., Full catastrophe living (revised edition): Using the wisdom of your body and mind to face stress, pain, and illness, 2013, Bantam Books; New York.

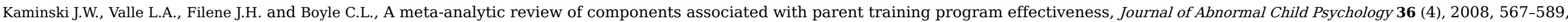
https://doi.org/10.1007/s10802-007-9201-9.

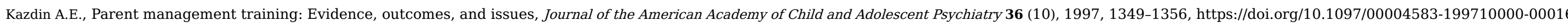
Kianifard F. and Islam M.Z., A guide to the design and analysis of small clinical studies, Pharmaceutical Statistics 10 (4), 2011, 363-368.

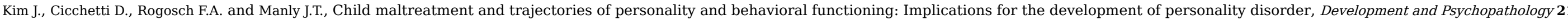
(3), 2009, 889-912, https://doi.org/10.1017/S0954579409000480.

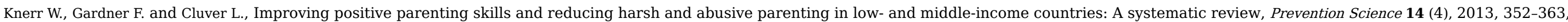
https://doi.org/10.1007/s11121-012-0314-1.

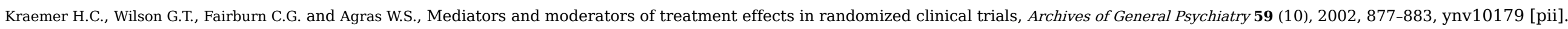

Kumkale G.T. and Albarracin D., The sleeper effect in persuasion: A meta-analytic review, Psychological Bulletin 130 (1), 2004, 143-172.

Kuo C., Operario D. and Cluver L., Depression among carers of AIDS-orphaned and other-orphaned children in Umlazi Township, South Africa, Global Public Health 7 (3), 2012, 253-269,

https://doi.org/10.1080/17441692.2011.626436.

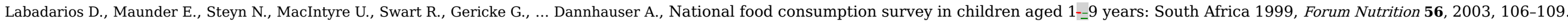

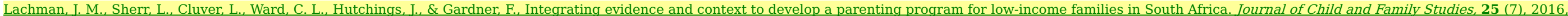

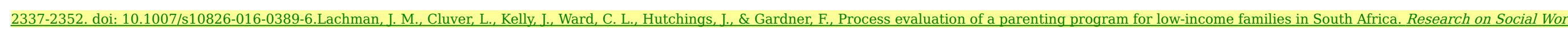

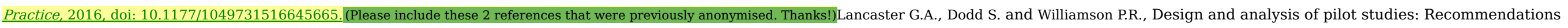
for good practice, Journal of Evaluation in Clinical Practice 10 (2), 2004, 307-312, https://doi.org/10.1111/j.2002.384.doc.x. 


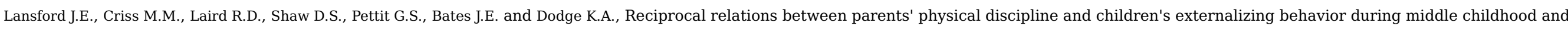
adolescence, Development and Psychopathology 23 (1), 2011, 225-238, https://doi.org/10.1017/S0954579410000751.

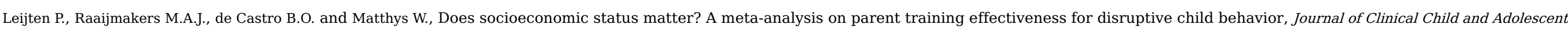
Psychology 42 (3), 2013, 384-392.

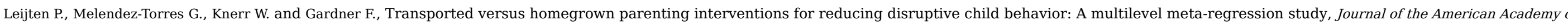
Child \& Adolescent Psychiatry 2016, https://doi.org/10.1016/j.jaac.2016.05.003.

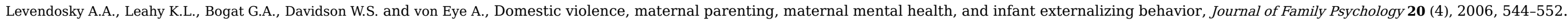

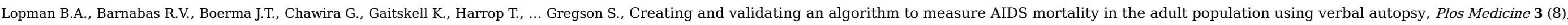
2006, 1273-1281, https://doi.org/10.1371/journal.pmed.0030312.

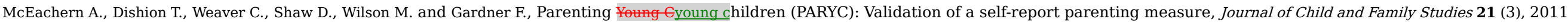
https://doi.org/10.1007/s10826-011-9503-y.

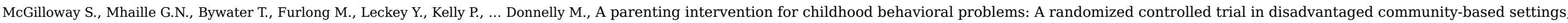
Journal of Consulting and Clinical Psychology 80 (1), 2012, 116.

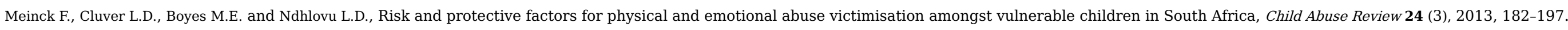

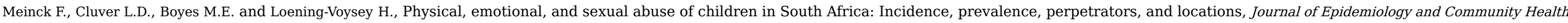
2016.

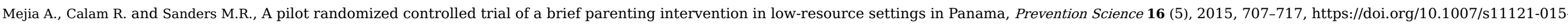
0551-1.

Mejia A., Leijten P., Lachman J.M. and Parra-Cardona J.R., Different strokes for different folks? Contrasting approaches to cultural adaptation of parenting interventions, Prevention Science 2016, https://doi.org/10.1007/s11121-016-0671-2.

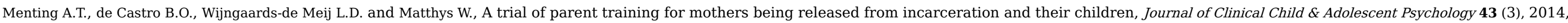
381-396.

Meth P., 'I don't like my children to grow up in this bad area': Parental anxieties about living in informal settlements, International Journal of Urban and Regional Research 37 (2), 2013, 537-555, https://doi.org/10.1111/J.1468-2427.2012.01199.X.

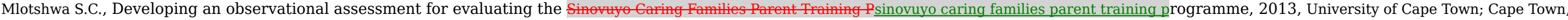
(Unpublished honours thesis)[Unpublished honours thesis].

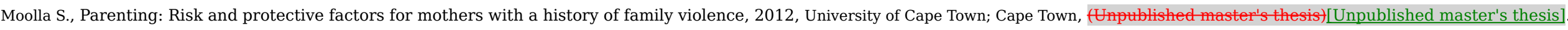

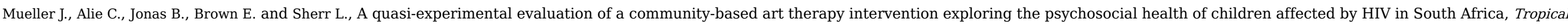
Medicine and International Health 16 (1), 2011, 57-66, https://doi.org/10.1111/J.1365-3156.2010.02682.X.

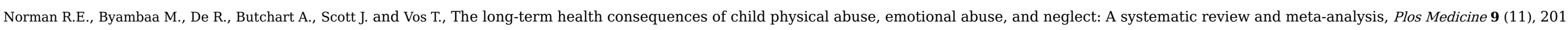
https://doi.org/10.1371/Journal.Pmed.1001349.

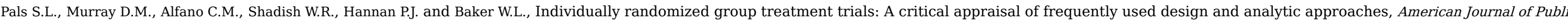




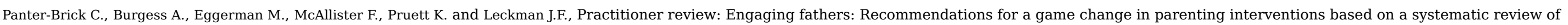
the global evidence, Journal of Child Psychology and Psychiatry 55 (11), 2014, 1187-1212, https://doi.org/10.1111/jcpp.12280.

Pardini D.A., Novel insights into longstanding theories of bidirectional parent-child influences: Introduction to the special section, Journal of Abnormal Child Psychology 36 (5), $2008,627-631$.

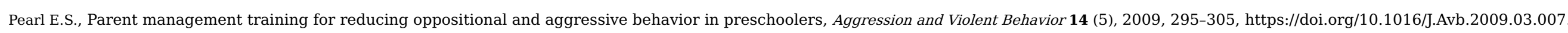

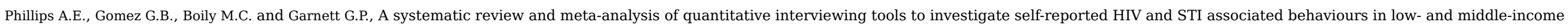

countries, International Journal of Epidemiology 39 (6), 2010, 1541-1555, https://doi.org/10.1093/ije/dyq114.

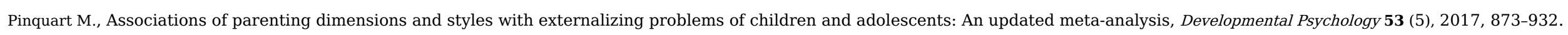

Potterton J., Stewart A. and Cooper P., Parenting stress of caregivers of young children who are HIV positive, African Journal of Psychiatry 10 (4), $2007,210-214$.

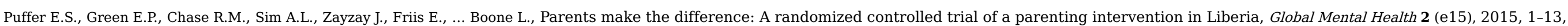

https://doi.org/10.1017/gmh.2015.12.

Sanders M.R., Waugh L., Tully L. and Hynes K., The Revised Family Observation Srevised family observation schedule. FOS-III, 1996, University of Queensland; Brisbane.

Statistics South Africa, Census 2011, 2012, Statistics South Africa; Pretoria.

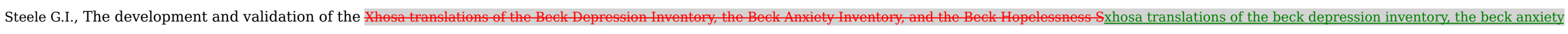

inventory, and the beck hopelessness scale, 2003, Grahamstown, South Africa; Rhodes University, (Unpublished master's thesis)[Unpublished master's thesis].

Straus M.A. and Douglas E.M., A short form of the Revised Conflict Tactics Scales, and typologies for severity and mutuality, Violence and Victims 19 (5), $2004,507-519$.

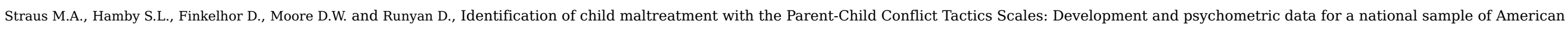
parents, Child Abuse \& Neglect 22 (4), 1998, 249-270, S0145-2134(97)00174-9[S0145-2134(97)00174-9].

Streiner D. and Geddes J., Intention to treat analysis in clinical trials when there are missing data, Evidence-Based Mental Health 4 (3), $2001,70-71$.

Thornberry T.P. and Henry K.L., Intergenerational continuity of maltreatment, J-A Child Psyolournal of Abnormal Child Psychology 41, 2013, 555, https://doi.org/10.1007/s10802-012-9697-5.

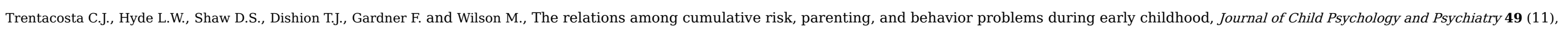
2008, 1211-1219, https://doi.org/10.1111/j.1469-7610.2008.01941.x.

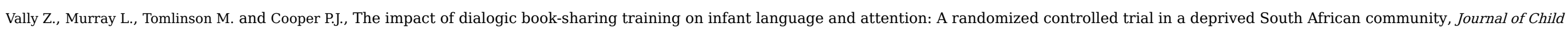
Psychology and Psychiatry 2014, https://doi.org/10.1111/jcpp.12352.

Wang R. and Ware J.H., Detecting moderator effects using subgroup analyses, Prevention Science 2011, https://doi.org/10.1007/s11121-011-0221-x.

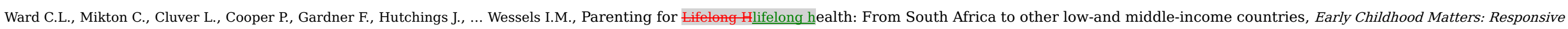
Parenting: A Strategy to Prevent Violence 2014, 49.

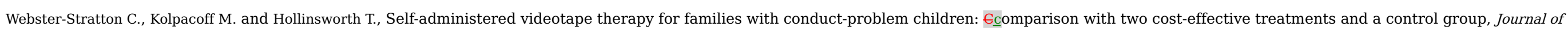
Consulting and Clinical Psychology 56 (4), 1988, 558-566.

Wessels I. and Ward C.L., A 'best buy' for violence prevention: Eevaluating parenting skills programmes, South African Crime Quarterly 54, 2015, 17-28, https://doi.org/10.4314/sacq.v54i1.2.

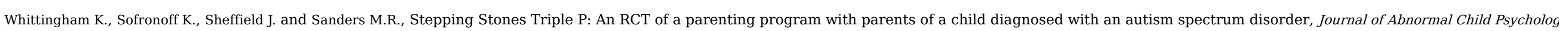


37 (4), 2009, 469-480.

Widom C.S. and Wilson H.W., Intergenerational transmission of violence, In: Lindert J. and Levav I., (Eds.), Violence and Mental Hmental health, 2015 , Springer; Dordrecht, 27-45.

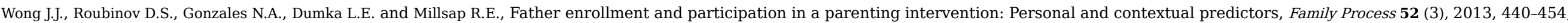
https://doi.org/10.1111/Famp.12024.

World Health Organization, Report on the consultation on child abuse prevention, 1999, World Health Organisation; Geneva, (Mareh 1999)[March 1999].

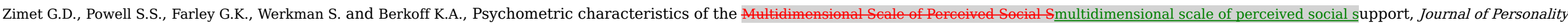

Assessment 55 (3-4), 1990, 610-617, https://doi.org/10.1080/00223891.1990.9674095.

\section{Footnotes}

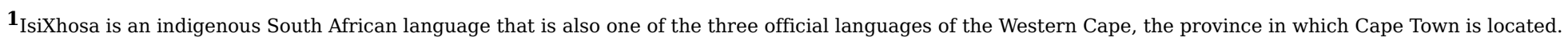

\section{Queries and Answers}

Query: Please check the dochead "Full Length Article", and correct if necessary.

Answer: Correct

Query: The author names have been tagged as given names and surnames (surnames are highlighted in teal color). Please confirm if they have been identified correctly.

Answer: Please change Inge M. Wessels to Inge Wessels

Query: Please check all author names and affiliations, and correct if necessary.

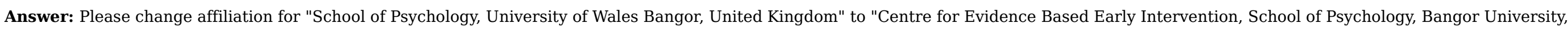
Wales"

Query: Please check the e-mail address that has been added for corresponding author "Jamie M. Lachman", and correct if necessary.

Answer: Added

Query: Please check the hierarchy of the section headings and correct if necessary.

Answer: Correct

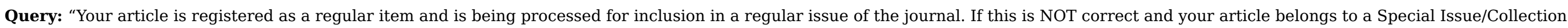
please contact a.srinivasaraghavan@elsevier.com immediately prior to returning your corrections."

Answer: Include as regular issue

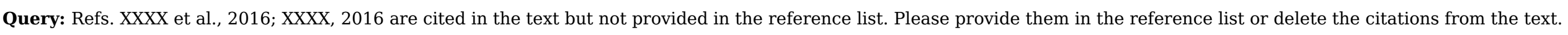

Answer: Added these references which were originally anonymised.

Query: Please note that Table 1 was not cited in the text. Please check that the citation added here in the appropriate place, and correct if necessary.

Answer: Please move Table 1 to before 3.1 Study flow header.

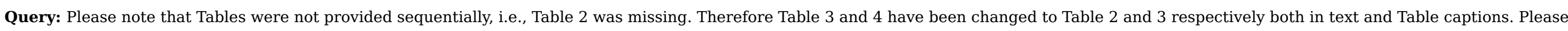
check and correct if necessary.

Answer: I have corrected these. The original reference to Table 1 was incorrect. 
Query: Please provide Table 4 along with table caption.

Answer: There is no longer a Table 4. This has been corrected.

Query: Please check the section funding and correct if necessary.

Answer: Correct

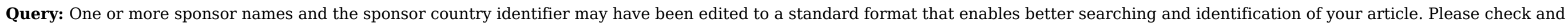
correct if necessary.

Answer: Yes

Query: Please provide the volume number/page number for Ref. "Hopkin et al., 2015; Leijten et al., 2016; Mejia et al., 2016; Vally et al., 2014".

Answer: Please use the following references:

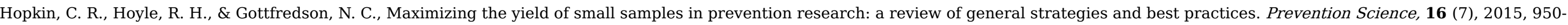
955; https://doi.org/10.1007/s11121-014-0542-7.

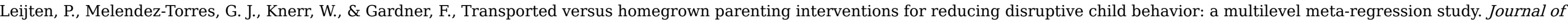
the American Academy of Child \& Adolescent Psychiatry, 55 (7), 2016, 610-617.

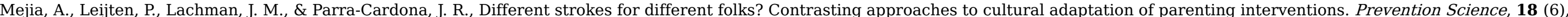
2017, 630-639, https://doi.org/10.1007/s11121-016-0671-2.

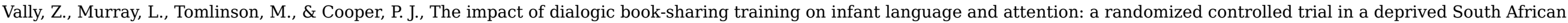
community. Journal of Child Psychology and Psychiatry, 56 (8), 865-873, https://doi.org/10.1111/jcpp.12352.

Query: Please provide the figure captions for Fig. 1 and 2.

Answer: Provided

Query: Please check the presentation of Tables 1-3 and correct if necessary.

Answer: Please move Table 2 to before 3.4 Outcomes section. Please move Table 3 to before 4 . Discussion 